\title{
PIK3CA mutation and methylation influences the outcome of colorectal cancer
}

\author{
SATORU IIDA $^{1}$, SHUNSUKE KATO ${ }^{1}$, MEGUMI ISHIGURO ${ }^{2}$, TAKATOSHI MATSUYAMA ${ }^{1}$, \\ TOSHIAKI ISHIKAWA ${ }^{3}$, HIROTOSHI KOBAYASHI ${ }^{4}$, TETSURO HIGUCHI ${ }^{2}$, \\ HIROYUKI UETAKE $^{3}$, MASAYUKI ENOMOTO ${ }^{1}$ and KENICHI SUGIHARA ${ }^{1}$ \\ ${ }^{1}$ Department of Surgical Oncology; ${ }^{2}$ Graduate Education Program for Cancer Treatment Specialists; \\ ${ }^{3}$ Department of Translational Oncology; ${ }^{4}$ Minimally Invasive Surgery Center, Graduate School, \\ Tokyo Medical and Dental University, Tokyo 113-8519, Japan
}

Received October 10, 2011; Accepted December 21, 2011

DOI: $10.3892 / \mathrm{ol} .2011 .544$

\begin{abstract}
Colorectal cancer (CRC) occurs through the accumulation of genetic and epigenetic alterations. The epigenetic abnormalities, in cooperation with genetic alterations, are capable of causing aberrant gene function that results in cancer. In the present study, we examined mutations and methylation status in $164 \mathrm{CRCs}$ to determine whether the combination of genetic and epigenetic alterations may be used to classify CRC patients in relation to their clinicopathological characteristics and outcomes. Mutation analyses for the KRAS and $P I K 3 C A$ genes were performed using direct sequencing, and the MethyLight method was used to determine the methylation status of BNIP3,pl6 and $h M L H 1$. The combination of the KRAS mutation with methylation status did not have any association with clinicopathological characteristics and outcomes. However, patients with the PIK3CA mutation and/ or high methylation ( 2 or 3 methylated genes) had significantly poorer outcomes in disease-specific survival (DSS) compared with those with wild-type PIK3CA and 0 or 1 methylated genes $(\mathrm{P}=0.0059)$. Additionally, multivariate analysis revealed that the PIK3CA mutation and/or a high level of methylation predicts a poor DSS independently of clinicopathological characteristics. Our results suggest that a combination of genetic and epigenetic alterations is a potent biomarker for predicting the prognosis of CRC.
\end{abstract}

\section{Introduction}

Colorectal cancer (CRC) is among the most common types of cancer in both males and females and is associated with

Correspondence to: Dr Satoru Iida, Department of Surgical Oncology, Graduate School, Tokyo Medical and Dental University, 1-5-45 Yushima, Bunkyo-ku, Tokyo, 113-8519, Japan

E-mail: s-iida.srg2@tmd.ac.jp

Key words: PIK3CA, BNIP3, methylation, mutation, colorectal cancer high mortality, particularly at advanced stages (1). Markers for defining individual risk signatures in CRC patients are of great clinical value, as they may allow for targeted therapies to improve the outcomes of CRC patients.

$\mathrm{CRC}$ arises through the accumulation of multiple genetic and epigenetic changes. Somatic mutations in $A P C, B R A F$, $K R A S, P I K 3 C A$ and TP53, as well as in other genes, have been frequently observed in CRC and are thought to drive colorectal tumorigenesis (2). We previously examined the mutation of genes in the EGFR pathway and found that the PIK3CA mutation is associated with poor prognosis in CRC patients $(8,11)$.

In addition to genetic alterations, epigenetic silencing is a prevalent mechanism by which abnormal gene inactivation may occur in cancer and is involved in initiation and promotion (3). A predominant mode of epigenetic alteration in cancer is gene silencing via the hypermethylation of the $\mathrm{CpG}$ island promoter. Hypermethylation results in abnormal silencing of a number of tumor suppressors in numerous human malignancies including CRC (3).

It has been suggested that epigenetic abnormalities are able to cooperate with genetic alterations to cause aberrant gene function and result in cancer (4). Thus, in the present study, we examined KRAS and PIK3CA mutations and the methylation status of BNIP3, pl6 and $h M L H 1$ and analyzed the correlation between these molecular alterations and the clinicopathological characteristics of CRC. We aimed to clarify whether a combination of genetic and epigenetic alterations could be used to classify CRC patients in relation to their clinicopathological characteristics and outcomes.

\section{Materials and methods}

Patients and tissue samples. After providing informed consent, a total of $165 \mathrm{CRC}$ patients who underwent surgical resection at Tokyo Medical and Dental University Hospital between March 2000 and April 2003 were recruited in this study. This study was approved by the institutional review board of the Tokyo Medical and Dental University. The study population (Table I) consisted of 62 females and 103 males, with a mean age of $64.5 \pm 10.3$ years (range, $37-89$ ). Sixty-two 


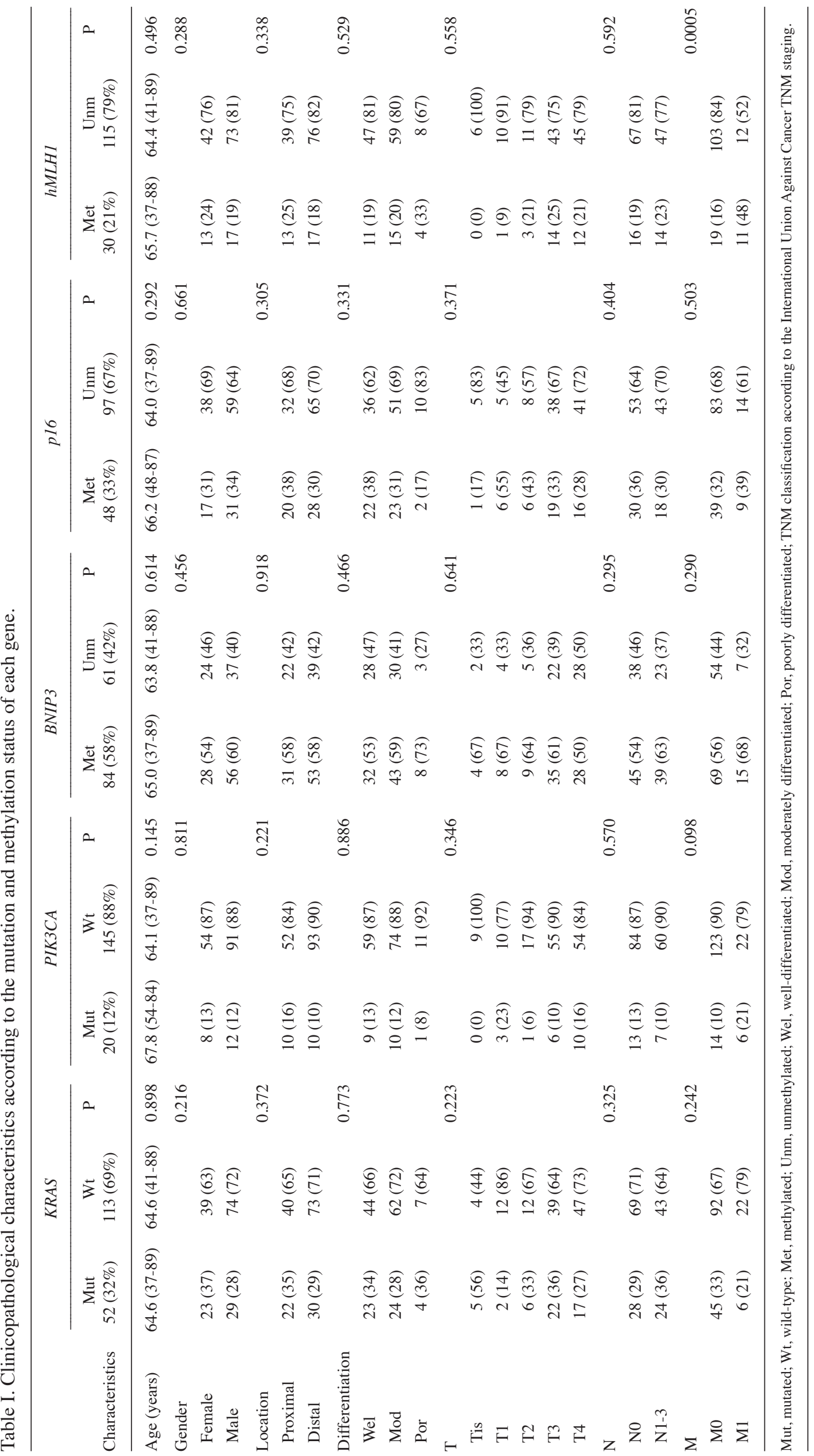


cancers were located in the proximal site and 103 were located in the distal site, including the rectum. Histological classification and tumor staging were performed according to the International Union Against Cancer Tumor-NodeMetastasis classification guidelines. No patient received preoperative chemotherapy or radiotherapy. Following surgery, patients with stage III CRC received oral or intravenous 5-fluorouracil (FU)-based adjuvant chemotherapy and patients with stage IV tumors received 5-FU-based systemic chemotherapy without radiotherapy. Patients were prospectively followed up following surgery for a median period of 64 months (range, 0.07-107.6). Resected specimens were fixed in $10 \%$ neutral buffered formalin and embedded in paraffin. For all cases, archival hematoxylin and eosin (H\&E) slides of primary tumors were retrieved and reviewed to confirm pathological features.

DNA extraction. Tissues were cut into $10-\mu \mathrm{m}$ sections from paraffin-embedded blocks. After specimens were deparaffinized and washed, tumor tissue was manually dissected for comparison with H\&E slides. The same amount of dissected tissue was used for each case. Genomic DNA was extracted using standard proteinase K (Invitrogen, Carlsbad, CA, USA) digestion, phenol/chloroform extraction, and ethanol precipitation, as previously described (5).

Mutation analysis. Exon 1 of the KRAS gene and exons 9 and 20 of the PIK3CA gene were selected for mutation analysis, since mutations are known to cluster in these regions $(6,7)$. Primer sequences and PCR conditions have been reported previously (8). Following the purification of PCR products using a Microcon YM-100 Centrifugal Filter (Millipore Corporation, Billerica, MA, USA) and Centri-Sep Columns (Princeton Separations, Adelphia, NJ, USA), direct sequencing was carried out using the Big Dye Terminator Cycle Sequencing kit (Applied Biosystems, Foster City, CA, USA) and analyzed using an Applied Biosystems 3130 Genetic Analyzer (Applied Biosystems). Somatic mutations were further validated by independent PCR amplification and sequencing and matched with normal mucosa.

MethyLight analysis. Sodium bisulfite conversion and DNA recovery was performed using EpiTect Bisulfite (Qiagen, Hilden, Germany). Following sodium bisulfite conversion, genomic DNA was analyzed using the MethyLight technique, a fluorescence-based real time PCR (Q-PCR) assay (9) and the ABI Prism 7300 Real Time PCR system (Taqman; Applied Biosystems). Four sets of primers and probes designed specifically for bisulfite-converted DNA were used. Three sets were used to detect methylation in the gene of interest and the remaining set served as a reference for $\beta$-actin (ACTB) to normalize for input DNA. Reference primers and probes were designed in a region of the $A C T B$ gene lacking $\mathrm{CpG}$ dinucleotides, thus allowing for equivalent amplification regardless of methylation levels. Primer and probe sequences were previously reported $(10,11)$. SssI-treated HCT-15 DNA was used as a fully methylated positive control (100\% methylation ratio). Parallel TaqMan PCR reactions were performed using primers specific for the bisulfite-converted methylated sequence of a particular locus and with $A C T B$ reference primers. In each case, triplicate threshold cycle $(\mathrm{Ct})$ values were obtained and averaged and expression levels were evaluated using the $2^{-\Delta \Delta \mathrm{Ct}}$ method. As an internal standard, each sample was normalized to its ACTB content and compared with the gene expression level of SssI-treated HCT-15 DNA as positive controls (calibration sample) as follows: $2^{\Delta \Delta \mathrm{Ct}}$ where $\Delta \Delta \mathrm{Ct}=(\mathrm{Ct}$-targetCt-reference) treated sample - (Ct-target-Ct-reference) calibrator sample. We defined the percentage of the fully methylated reference (PMR) to be $2^{\Delta \Delta \mathrm{Ct}} \times 100 \%$. To define pl6 or $h M L H 1$ methylation status, a PMR cut-off value of 4 was used based on previously validated data (9). Based on the distribution of PMR values in normal colon epithelial tissue (10), a PMR cut-off value of $>0$ was used to define positive methylation status for BNIP3.

Statistical analysis. Statistical analysis was carried out using StatView Software (version 5.0). To estimate the differences among the groups, the Chi-square test, Fisher's exact test, Student's t-test and log-rank test were used where appropriate. The Kaplan-Meier method was used to estimate survival. Survival was calculated beginning from the date of surgery. Prognostic factors were estimated using multivariate analysis and the Cox proportional hazards model. $\mathrm{P}<0.05$ was considered to indicate a statistically significant result.

\section{Results}

Mutation of KRAS and PIK3CA and methylation of BNIP3, p16 and $h M L H 1$. KRAS mutations in exon 1 were observed in $32 \%$ of cases and PIK3CA mutations in exons 9 and 20 in $12 \%$ of cases. Methylation frequencies of the examined genes were $58 \%$ for BNIP3, $33 \%$ for p16 and $21 \%$ for $h M L H 1$. Although $h M L H 1$ methylation was significantly associated with distant metastasis $(\mathrm{P}=0.0005)$, other associations were not observed between clinicopathological characteristics and the mutation or methylation status of individual genes (Table I).

Correlation between mutation and methylation. Associations among gene mutations and gene methylations are shown in Table II. The KRAS mutation was significantly associated with BNIP3 $(\mathrm{P}<0.0001)$ and pl6 methylation $(\mathrm{P}=0.04)$ as well as with the number of methylated genes $(\mathrm{P}=0.0082)$. p16 methylation was associated with BNIP3 methylation $(\mathrm{P}<0.0001)$ and $h M L H 1$ methylation $(\mathrm{P}=0.03)$.

Correlation between molecular markers and outcomes. The correlation between the molecular parameters and diseasespecific survival (DSS) were analyzed (Table III). Patients with $h M L H 1$-methylated tumors had significantly shorter DSS compared with those without methylation [hazards ratio (HR), $2.231 ; \mathrm{P}=0.05]$, but mutation or methylation of other single genes did not affect DSS. Having mutations in both the KRAS and PIK3CA genes did not correlate with DSS. By contrast, when cases were divided into 2 groups, namely a low methylation group ( 0 or 1 methylated genes) and a high methylation group ( 2 or 3 methylated genes), the high methylation group had significantly shorter DSS than the low methylation group (HR, 2.681; $\mathrm{P}=0.01)$. Integration of the KRAS mutation and methylation status of multiple genes did not reveal an association with DSS. However, CRCs were divided into 4 groups: 
Table II. Concordance between the mutation and methylation status of each gene.

\begin{tabular}{|c|c|c|c|c|c|c|c|c|c|c|}
\hline & \multicolumn{2}{|c|}{ PIK3CA } & \multicolumn{2}{|c|}{ BNIP3 } & \multicolumn{2}{|c|}{ p16 } & \multicolumn{2}{|c|}{$h M L H 1$} & \multicolumn{2}{|c|}{ Methylation $^{\mathrm{a}}$} \\
\hline & Mut & $\mathrm{Wt}$ & Met & Unm & Met & Unm & Met & Unm & High & Low \\
\hline$K R A S$ & \multicolumn{2}{|c|}{$\mathrm{P}=0.918$} & \multicolumn{2}{|c|}{$\mathrm{P}<0.0001$} & \multicolumn{2}{|c|}{$\mathrm{P}=0.0402$} & \multicolumn{2}{|c|}{$\mathrm{P}=0.467$} & \multicolumn{2}{|c|}{$\mathrm{P}=0.0082$} \\
\hline Mut & 7 & 45 & 39 & 9 & 21 & 26 & 8 & 39 & 24 & 23 \\
\hline $\mathrm{Wt}$ & 13 & 88 & 45 & 52 & 27 & 71 & 22 & 77 & 28 & 70 \\
\hline PIK3CA & & & \multicolumn{2}{|c|}{$\mathrm{P}=0.827$} & \multicolumn{2}{|c|}{$\mathrm{P}=0.982$} & \multicolumn{2}{|c|}{$\mathrm{P}=0.428$} & \multicolumn{2}{|c|}{$\mathrm{P}=0.775$} \\
\hline Mut & & & 10 & 8 & 6 & 12 & 5 & 13 & 7 & 11 \\
\hline $\mathrm{Wt}$ & & & 74 & 53 & 42 & 85 & 25 & 102 & 45 & 82 \\
\hline BNIP3 & & & & & \multicolumn{2}{|c|}{$\mathrm{P}<0.0001$} & \multicolumn{2}{|c|}{$\mathrm{P}=0.541$} & & \\
\hline Met & & & & & 42 & 39 & 19 & 64 & & \\
\hline Unm & & & & & 4 & 54 & 11 & 48 & & \\
\hline pl6 & & & & & & & \multicolumn{2}{|c|}{$\mathrm{P}=0.0295$} & & \\
\hline Met & & & & & & & 15 & 33 & & \\
\hline Unm & & & & & & & 15 & 81 & & \\
\hline
\end{tabular}

${ }^{a}$ Methylation high, 2 or 3 methylated genes; low, 0 or 1 methylated genes. Mut, mutated; Wt, wild-type; Met, methylated; Unm, unmethylated.

Table III. Correlation of molecular parameters with DSS.

\begin{tabular}{|c|c|c|c|c|}
\hline & & HR & $95 \% \mathrm{CI}$ & $\mathrm{P}$ \\
\hline$K R A S$ & (Mutant vs. wild) & 0.930 & $0.418-2.071$ & 0.8590 \\
\hline$P I K 3 C A$ & (Mutant vs. wild) & 1.429 & $0.540-3.779$ & 0.4721 \\
\hline BNIP3 & (Methylated vs. unmethylated) & 2.233 & $0.944-5.284$ & 0.0675 \\
\hline p16 & (Methylated vs. unmethylated) & 1.950 & $0.912-4.169$ & 0.0849 \\
\hline$h M L H 1$ & (Methylated vs. unmethylated) & 2.231 & $1.000-4.977$ & 0.0500 \\
\hline Mutation $^{\mathrm{a}}$ & (Mutant vs. wild) & 1.001 & $0.468-2.139$ & 0.9983 \\
\hline Methylation $^{\mathrm{b}}$ & (High vs. low) & 2.681 & $1.253-5.747$ & 0.0110 \\
\hline Mutation/methylation & (Mutant and/or high vs. wild and low) & 1.499 & $0.634-3.546$ & 0.3569 \\
\hline KRAS/methylation & (Mutant and/or high vs. others) & 1.675 & $0.766-3.663$ & 0.1963 \\
\hline PIK3CA/methylation & (Mutant and/or high vs. others) & 2.924 & $1.312-6.494$ & 0.0087 \\
\hline
\end{tabular}

${ }^{a}$ Mutant, at least one mutation gene; wild, no mutation. ${ }^{b}$ Methylation high, 2 or 3 methylated genes; low, 0 or 1 methylated genes. CI, confidence interval; HR, hazards ratio.

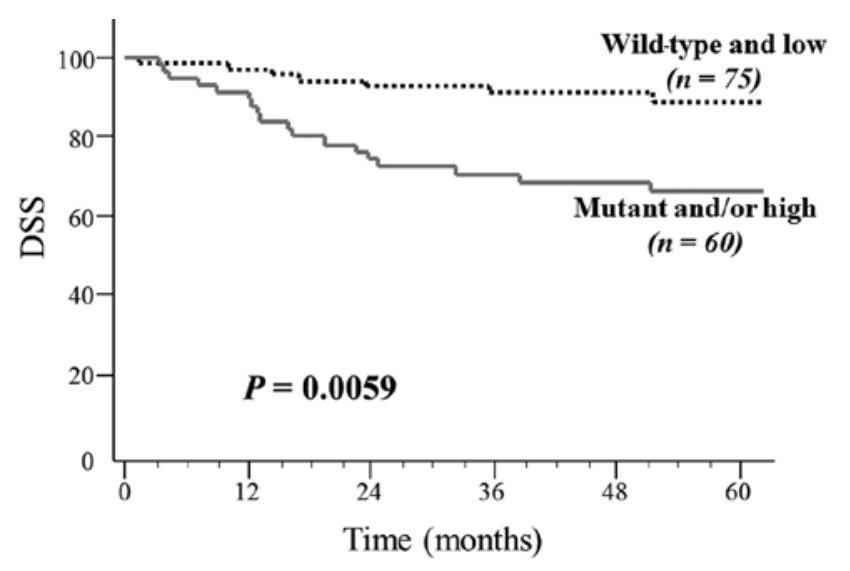

Figure 1. Kaplan-Meier estimate of DSS according to PIK3CA mutation and methylation classification. DSS, disease-specific survival.
CRC containing i) PIK3CA mutation and high methylation (5.2\%); ii) PIK3CA mutation and low methylation (8.1\%); iii) no mutation and high methylation (31.1\%); and iv) no mutation and low methylation (55.6\%). Patients with the PIK3CA mutation and/or high methylation (groups i, ii and iii) had a significantly poorer outcome in DSS compared with those with wild-type PIK3CA and low methylation (group iv; HR, 2.924; $\mathrm{P}=0.0087)(\mathrm{P}=0.0059$, log-rank test; Fig. 1$)$.

Results of the univariate and multivariate analyses using the Cox proportional hazards model are shown in Table IV. Univariate analysis revealed that the PIK3CA mutation and/or high methylation was a significant prognostic factor for DSS, when used with the depth of the tumor, lymph node metastasis, distant metastasis, histological differentiation, lymphatic invasion and vessel invasion. Variables with $\mathrm{P}<0.05$ in the 
Table IV. Cox proportional hazards model of prognostic factors of DSS.

\begin{tabular}{|c|c|c|c|c|c|c|c|}
\hline \multirow[b]{2}{*}{ Characteristics } & & \multicolumn{3}{|c|}{ Univariate analysis } & \multicolumn{3}{|c|}{ Multivariate analysis } \\
\hline & & $\mathrm{HR}$ & $95 \% \mathrm{CI}$ & $P$ & HR & $95 \% \mathrm{CI}$ & $\mathrm{P}$ \\
\hline Gender & (Female vs. male) & 0.424 & $0.171-1.050$ & 0.0636 & & & \\
\hline Age & $(\geq 65$ vs. $<65)$ & 0.699 & $0.328-1.487$ & 0.3526 & & & \\
\hline Location & (Distal vs. proximal) & 0.839 & $0.389-1.808$ & 0.6539 & & & \\
\hline $\mathrm{T}$ & $(\mathrm{T} 1,2$ vs. $\mathrm{T} 3,4)$ & 8.547 & $1.157-62.50$ & 0.0354 & 2.451 & $0.270-22.20$ & 0.4253 \\
\hline $\mathrm{N}$ & $(\mathrm{N}-$ vs. $\mathrm{N}+)$ & 3.559 & $1.558-8.130$ & 0.0026 & 1.368 & $0.504-3.717$ & 0.5377 \\
\hline M & (M0 vs. M1) & 7.874 & $3.448-17.86$ & $<0.0001$ & 7.092 & $2.732-18.18$ & $<0.0001$ \\
\hline Differentiation & (Por vs. wel/mod) & 3.031 & $1.046-8.781$ & 0.0411 & 0.611 & $0.172-2.172$ & 0.4466 \\
\hline Ly & (Ly0,1 vs. ly 2,3$)$ & 4.831 & $2.247-10.31$ & $<0.0001$ & 3.891 & $1.379-10.99$ & 0.0102 \\
\hline $\mathrm{v}$ & (v0,1 vs. v2,3) & 4.049 & $1.399-11.76$ & 0.0099 & 1.761 & $0.540-5.747$ & 0.3482 \\
\hline PIK3CA/methylation & $\begin{array}{l}\text { (Mutant and/or high } \\
\text { vs. others) }\end{array}$ & 2.924 & $1.312-6.494$ & 0.0087 & 2.793 & $1.220-6.410$ & 0.0151 \\
\hline
\end{tabular}

HR, hazards ratio; CI, confidence interval; TNM classification according to the International Union Against Cancer TNM staging; por, poorly differentiated; wel, well-differentiated; mod, moderately differentiated; ly, lymphatic invasion; v, vessel invasion; high methylation, 2 or 3 methylated genes.

univariate analysis were used for the multivariate analysis. PIK3CA mutation and/or high methylation, as well as distant metastasis and lymphatic invasion, were found to be independent and significant prognostic factors for DSS.

\section{Discussion}

In this study, we examined mutations in KRAS and PIK3CA, which comprise components of the EGFR pathway, and the methylation status of BNIP3, pl6 and $h M L H 1$ in CRCs to evaluate patients with the PIK3CA mutation and/or 2 or more methylations with a poorer prognosis compared with other patients. Our results suggest that a combination of epigenetic and genetic alterations is a potent biomarker for predicting CRC prognosis.

Integrated analysis of epigenetic and genetic alterations is increasingly significant in cancer research. Since CRC develops as a result of accumulating genetic and epigenetic alterations, the combination of genetic and epigenetic profiles could confer differential clinical phenotypes and potential variability in survival. Ward et al reported that although DNA methylation is associated with a worse outcome in CRC patients, this adverse prognostic effect is lost in methylated tumors with microsatellite instability (12). By integrating genetic and epigenetic analyses, Shen et al revealed that colon cancers correspond to 3 molecularly distinct subclasses of disease, each of which is relatively homogeneous (13). In the present study, CRCs were divided into 4 groups according to PIK3CA mutations and the number of methylated genes: CRCs with i) PIK3CA mutation and 2 or 3 methylations (5.2\%); ii) PIK3CA mutation and less than 2 methylations (8.1\%); iii) no mutation and 2 or 3 methylations (31.1\%); and iv) no mutations and less than 2 methylations (55.6\%). The last group was associated with better prognosis than the former 3 groups. CRC patients with the PIK3CA mutation and/or more than 1 methylation had worse outcomes compared with the other groups. This finding suggests that mutation analysis and deter- mining methylation status may provide significant predictive information regarding tumor behavior and clinical outcome in CRC patients.

BNIP3 is a pro-apoptotic member of the Bcl-2 family (14). The expression of BNIP3 is induced by hypoxia, such as that which occurs during cardiac ischemia and in the hypoxic regions of tumors, and it acts against pro-survival proteins, including Bcl-2 and Bcl-xl (15-18). Transcriptional silencing of the BNIP3 gene has recently been observed in multiple human cancer cell lines, including colorectal, gastric, pancreatic, and hematopoietic tumors (19). Murai et al reported that aberrant hypermethylation of BNIP3 was detected in $66 \%$ of primary colorectal and $49 \%$ of primary gastric cancers, but not in normal tissue; these authors also revealed that BNIP3 is silenced by DNA methylation (20). Akada et al and Erkan et al revealed that loss of BNIP3 expression correlates with poor prognosis and increased chemoresistance in patients with pancreatic cancer $(21,22)$. Manka et al demonstrated that $B N I P 3$ silencing induces or increases the metastatic growth of breast cancer in distant organs (23). Moreover, our previous study also indicated that BNIP3 methylation is associated with poor clinical outcome and chemoresistance in primary CRC (10). These previous studies suggest that reduced BNIP3 expression contributes to carcinogenesis of the colon and rectum and may be a predictive factor for the prognosis of CRC. Therefore, the BNIP3 gene, as well as the $h M L H 1$ and p16 genes, were tested in our methylated gene sets for predicting the outcome of CRC.

Activation of the phosphatidylinositol3-kinase (PI3K)/AKT pathway is thought to be critical in CRC development and clinical behavior $(6,8,24,25)$. PIK3CA encodes the $\mathrm{p} 110 \alpha$ catalytic subunit of PI3K and is mutated in $10-32 \%$ of CRCs $(6,8,24,26-29)$. PIK3CA mutations elevate kinase activity, thereby activating the PI3K/AKT pathway and contributing to tumorigenesis through decreased apoptosis, loss of contact inhibition and increased tumor invasion $(6,30)$; these factors have been associated with poor outcomes in CRC 
patients $(8,24,31)$. Although previous studies have investigated the role of the PIK3CA mutation and gene methylation in CRC, the data are inconclusive. Certain studies reported a significantly higher frequency of the PIK3CA mutation in the $\mathrm{CpG}$ island methylator phenotype (CIMP)-high tumor compared with CIMP-negative tumors and a significant correlation between the PIK3CA mutation and RASSF2 gene methylation $(29,32,33)$, whereas other studies failed to observe this correlation $(11,34)$. In the present study, although CIMP status was not examined, the PIK3CA mutation did not exhibit an association with the methylation status of the genes examined. Moreover, few studies have described the effect of the combination of the PIK3CA mutation with gene methylation on patient outcome. Ogino et al demonstrated that the effect of the PIK3CA mutation on CRC patient survival is not significantly modified by CIMP (31). Their result was inconsistent with our finding that integration of the PIK3CA mutation and methylation status may be used to predict outcomes for CRC patients. It is possible that the methylated gene set used in this study was different from that used in the Ogino study. The BNIP gene included in our methylated set was not included in CIMP markers used to date. Moreover, there have been no studies regarding the influence of integrating the $P I K 3 C A$ mutation and methylation status of the BNIP3 gene on outcomes of CRC. It would be useful to examine whether mutating other genes from the EGFR pathway also affects CRC prognosis in cooperation with BNIP3 methylation.

In conclusion, by integrating genetic and epigenetic alterations, we revealed that CRC patients with poor prognosis could be identified. Further studies are necessary to confirm our finding that a combination of the PIK3CA mutation with gene methylation may be useful for predicting outcomes of CRC patients and to elucidate the underlying mechanism behind these findings.

\section{References}

1. Jemal A, Siegel R, Xu J and Ward E: Cancer statistics. CA Cancer J Clin 60: 277-300, 2010.

2. Wood LD, Parsons DW, Jones S, et al: The genomic landscape of human breast and colorectal cancers. Science 318: 1108-1113, 2007.

3. Jones PA and Baylin SB: The fundamental role of epigenetic events in cancer. Nat Rev Genet 3: 415-428, 2002.

4. Jones PA and Baylin SB: The epigenomics of cancer. Cell 128: 683-692, 2007.

5. Iida S, Akiyama Y, Nakajima T, Ichikawa W, Nihei Z, Sugihara K, and Yuasa Y: Alterations and hypermethylation of the p14ARF gene in gastric cancer. Int J Cancer 87: 654-658, 2000.

6. Samuels Y, Wang Z, Bardelli A, et al: High frequency of mutations of the PIK3CA gene in human cancers. Science 304: $554,2004$.

7. Vogelstein B, Fearon ER, Hamilton SR, et al: Genetic alterations during colorectal-tumor development. N Engl J Med 319: 525-532, 1988

8. Kato S, Iida S, Higuchi T, et al: PIK3CA mutation is predictive of poor survival in patients with colorectal cancer. Int J Cancer 121: 1771-1778, 2007.

9. Eads CA, Danenberg KD, Kawakami K, et al: MethyLight: a high-throughput assay to measure DNA methylation. Nucleic Acids Res 28: E32-00, 2000.
10. Shimizu S, Iida S, Ishiguro M, et al: Methylated BNIP3 gene in colorectal cancer prognosis. Oncol Lett 1: 865-872, 2010.

11. Aoyagi H, Iida S, Uetake H, et al: Effect of classification based on combination of mutation and methylation in colorectal cancer prognosis. Oncol Rep 25: 789-794, 2011.

12. Ward RL, Cheong K, Ku SL, Meagher A, O'Connor T and Hawkins NJ: Adverse prognostic effect of methylation in colorectal cancer is reversed by microsatellite instability. J Clin Oncol 21: 3729-3736, 2003.

13. Shen L, Toyota M, Kondo Y, et al: Integrated genetic and epigenetic analysis identifies three different subclasses of colon cancer. Proc Natl Acad Sci USA 104: 18654-18659, 2007.

14. Reed JC: Bcl-2 family proteins. Oncogene 17: 3225-3236, 1998.

15. Labi V, Erlacher M, Kiessling S and Villunger A: BH3-only proteins in cell death initiation, malignant disease and anticancer therapy. Cell Death Differ 13: 1325-1338, 2006.

16. Strasser A: The role of BH3-only proteins in the immune system. Nat Rev Immunol 5: 189-200, 2005.

17. Willis SN and Adams JM: Life in the balance: how BH3-only proteins induce apoptosis. Curr Opin Cell Biol 17: 617-625, 2005.

18. Vande VC, Cizeau J, Dubik D, et al: BNIP3 and genetic control of necrosis-like cell death through the mitochondrial permeability transition pore. Mol Cell Biol 20: 5454-5468, 2000.

19. Mellor HR and Harris AL: The role of the hypoxia-inducible BH3-only proteins BNIP3 and BNIP3L in cancer. Cancer Metastasis Rev 26: 553-566, 2007.

20. Murai M, Toyota M, Suzuki H, et al: Aberrant methylation and silencing of the BNIP3 gene in colorectal cancer and gastric cancer. Clin Cancer Res 11: 1021-1027, 2005.

21. Akada M, Crnogorac-Jurcevic T, Lattimore S, et al: Intrinsic chemoresistance to gemcitabine is associated with decreased expression of BNIP3 in pancreatic cancer. Clin Cancer Res 11: 3094-3101, 2005.

22. Erkan M, Kleeff J, Esposito I, et al: Loss of BNIP3 expression is a late event in pancreatic cancer contributing to chemoresistance and worsened prognosis. Oncogene 24: 4421-4432, 2005.

23. Manka D, Spicer Z and Millhorn DE: Bcl-2/adenovirus E1B $19 \mathrm{kDa}$ interacting protein-3 knockdown enables growth of breast cancer metastases in the lung, liver, and bone. Cancer Res 65: 11689-11693, 2005.

24. Barault L, Veyrie N, Jooste V, et al: Mutations in the RAS-MAPK, PI(3)K (phosphatidylinositol-3-OH kinase) signaling network correlate with poor survival in a population-based series of colon cancers. Int J Cancer 122: 2255-2259, 2008.

25. Manning BD and Cantley LC: AKT/PKB signaling: Navigating downstream. Cell 129: 1261-1274, 2007.

26. Velho S, Oliveira C, Ferreira A, et al: The prevalence of PIK3CA mutations in gastric and colon cancer. Eur J Cancer 41: 1649-1654, 2005.

27. Benvenuti S, Frattini M, Arena S, et al: PIK3CA cancer mutations display gender and tissue specificity patterns. Hum Mutat 29: 284-288, 2008.

28. Abubaker J, Bavi P, Al-Harbi S, et al: Clinicopathological analysis of colorectal cancers with PIK3CA mutations in Middle Eastern population. Oncogene 27: 3539-3545, 2008.

29. Nosho K, Kawasaki T, Ohnishi M, et al: PIK3CA mutation in colorectal cancer: relationship with genetic and epigenetic alterations. Neoplasia 10: 534-541, 2008.

30. Samuels Y, Diaz LA Jr, Schmidt-Kittler O, et al: Mutant PIK3CA promotes cell growth and invasion of human cancer cells. Cancer Cell 7: 561-573, 2005.

31. Ogino S, Nosho K, Kirkner GJ, et al: PIK3CA mutation is associated with poor prognosis among patients with curatively resected colon cancer. J Clin Oncol 27: 1477-1484, 2009.

32. Nosho K, Yamamoto H, Takahashi T, et al: Genetic and epigenetic profiling in early colorectal tumors and prediction of invasive potential in pT1 (early invasive) colorectal cancers. Carcinogenesis 28: 1364-1370, 2007.

33. Whitehall V, Rickman C, Bond C, et al: Oncogenic PIK3CA mutations in colorectal cancers and polyps. Int J Cancer: 2011 (Epub ahead of print) doi:10.1022/ijc.26440

34. Bruin SC, He Y,Mikolajewska-Hanclich I, et al: Molecular alterations associated with liver metastases development in colorectal cancer patients. Br J Cancer 105: 281-287, 2011. 